autor, para los conceptos morales. $\mathrm{El}$ problema que consiste en saber qué son en sí mismos lo bueno, lo bello, lo santo o lo justo no tiene sentido, ya que no disponemos de ningún criterio para verificar los correspondientes juicios. Lo único sensato que al respecto se ha dicho ha procedido siempre de los "razonamientos inductivos" de Sócrates, "que consistían en descubrir lo que los griegos de la época llamaban en la práctica una conducta justa o una obra bella" (pág. 417). En resumen: podemos investigar qué es lo que en determinada sociedad o en una cierta época se ha considerado como valioso, mas no tiene sentido inquirir qué es valioso en sí.

Aun cuando Rougier sostiene que "la nueva teoría del conocimiento" excluye todo "ejemplarismo" y todo "realismo ontológico", el lector no puede dejar de preguntarse cómo es posible tan absoluta exclusión si se sostiene que la verdad de los juicios consiste "en la correspondencia de un sistema de simbolos y de relaciones entre éstos con los objetos a que se les refiere y las relaciones de tales objetos". ¿No equivale esto a reconocer que la correspondencia entre los símbolos y los objetos, o entre las relaciones simbólicas y objetivas no depende de la elección de los símbolos, de los axiomas y, en general, de los esquemas empleados por el investigador? ¿Cómo entender que los conocimientos científicos no sólo nos permitan explicar la realidad, sino poner las fuerzas de ésta al servicio de nuestros propósitos, si entre las estructuras del conocimiento, las del ser ideal y las del ser real no hay al menos, como dice Nicolai Hartmann, una parcial coincidencia?

Pese a las objeciones que desde el punto de vista de otras posturas filosóficas puedan hacerse a Rougier, cualquier lector sin prejuicios tendrá que admitir que su obra, tanto por la riqueza de la información como por la fuerza dialéctica que el autor desplie- ga, es un excelente alegato en favor de esa corriente de pensamiento que en la filosofía contemporánea se conoce con el nombre de neo-positivismo vienés.

Eduardo García Máynez

Fenomenología del conocimiento. El problema de la constitución del objeto en la filosofía de Husserl, por Ernesto Mayz Vallenilla. Facultad de Humanidades y Educación. Universidad Central de Venezuela, Caracas, 1956.

Las dos ideas fundamentales de la Fenomenología, decía Eugen Fink, son la reducción fenomenológica y la teoría. de la constitución. Por la primera resulta inseparable del idealismo trascendental, por la segunda implica una teoría del conocimiento fundante de la objetividad. Esta concepción del más fiel de los discípulos de Husserl, podría definir el criterio que preside la interpretación de Mayz Vallenilla. La Fenomenología no es escindible en su "parteblanca" y su "parte negra" como algunos comentaristas, detenidos en las. primicias del método, quisieran creer. La descripción eidética de las primeras obras husserlianas no constituía aún el' método pleno, y sólo fue, para Husserl, una vía de acceso a la actitud trascendental; si queremos entender su filosofía, debemos partir, pues, de la reducción fenomenológico-trascendental. Por otra parte, la Fenomenología es una teoría del conocimiento en la cual el proble-ma de lo dado implica el de lo constituído; la teoría de la constitución pone bajo. su verdadera luz los anteriores pasos metódicos. Ésta es la posición que Husserl, desde las Ideas, no abandonará. Y es la posición en que Mayz Vallenilla: se coloca para juzgar la Fenomenología. Si su libro convence es porque está organizado según el criterio interpre- 
tativo justo. Justo, aquí, vale tanto como fiel. Mayz Vallenilla es un intérprete fiel en todo momento: no sólo en el cuidadoso cotejo de los textos o en la prudencia con que restringe su juicio a lo que ellos permiten, sino también en el rigor que impone a su investigación, desdeñosa de concesiones y afanosa de precisión. Su prosa resulta, por ello, a menudo dura. Entre el rigor y la agilidad de exposición, Mayz se decidió - como lo exigía su temapor el primero. $\mathrm{Y}$ si el avance del discurso es lento, prolijo a veces, alcanza en cambio valores superiores: la precisión del análisis, la veracidad de la interpretación. Disciplina, ascesis filosófica, necesaria en todo pensamiento; imprescindible si de Husserl se trata:

El subtítulo del libro revela mejor que el título (a todas luces demasiado amplio) el tema tratado. Estamos frente a una exposición de la doctrina acerca de la constitución del objeto en el primer tomo de las Ideas relativas a una fenomenología pura y una filosofía fenomenológica. Por haber sido publicados los tomos siguientes de las Ideas después de iniciada la investigación, no pudo el autor estudiarlos. Lo cual deja al margen de la exposición temas tan importantes para la constitución como la temporalidad y la intersubjetividad. Creemos que algunas indicaciones de libros anteriores (las Vorlesungen zur Phaenomenologie des inneren Zeitbewusstseins de 1928, o incluso las Meditaciones Cartesianas y la Formale und transzendentale Logik) que el autor menciona y utiliza, hubieran podido aprovecharse para no silenciar enteramente esos problemas. Con todo, el primer tomo de las Ideas puede bastar para el estudio de los primeros pasos en la doctrina y servir de base para una introducción al tema; y ésa es jus. tamente la pretensión del autor (pág. 368).

El comentario sigue un procedimiento general: la explicitación de los principios que vertebran la reflexión filo. sófica o que expresan sus resultados. Se logra, así, ordenar en forma sistemática las prolijas meditaciones husserlianas, sin salir de su propio pensamiento.

La Introducción muestra el lugar del tema de la constitución en el método. El sentido del método es la crítica del conocimiento dirigida a instaurar la fi. losofía como ciencia rigurosa. Por ello no puede soslayar la pregunta por la conciencia como primer fundamento sin supuestos ni eludir el recurso a lo trascendental. El autor se coloca, de golpe, en la perspectiva inaugurada por la Filosofía como ciencia rigurosa, rechazando con Husserl la posibilidad de separar el método fenomenológico y la filosofía trascendental. Ahora bien, el problema de la constitución del objeto es "el paso más decisivo y fundamental que era necesario a la Fenomenología para realizar su máxima aspiración: la de convertirse en el modelo de una ciencia rigurosa" (pág. 58).

El Capitulo I empieza planteando el problema en términos sugerentes: ¿Cómo algo trascendente puede ser conocido en la inmanencia de la conciencia? " ¿Se entiende sin más, cómo un término en principio extraño puede venir a ser intraño a la conciencia? ¿Se comprende claramente cuál es la transformación que debe sufrir 'algo' no ingrediente para llegar a ser parte esen. cial de la conciencia y con eso ser, a su vez, consciente?" (pág. 87). ¿Qué es ese "algo"? La psicología empírica es incapaz de dar una respuesta, de alli la necesidad del método. Desde el principio, liga el autor la crítica del psicologismo y la subsecuente formulación del método con el problema de la constitución. Se restituye, así, al método fenomenológico su coherencia interna, que otras interpretaciones más intencionadas le habian querido arrebatar. Sigue una exposición de las reducciones. La distinción entre reducción eidética y reducción trascendental es la más precisa y clara que conozcamos. La prioridad que el autor parece con- 
ceder a la segunda sobre la primera responde al enfoque de las Ideas y arroja su verdadera luz sobre el sentido que tuvo el método eidético en las obras antericres. Con todo, parece contradecir la anterioridad cronológica de la reducción eidética en la evolución del pensamiento husserliano y merecería aquí alguna aclaración.

La reducción nos enfrenta con las estructuras fundamentales de la conciencia pura. En particular, debemos dirigirnos al nóema como lugar en que se encierra la referencia objetiva. El Capitulo II pregunta por los elementos componentes del correlato noemático. Análisis apretado de sus estructuras. Quedan ordenados, con claridad y precisión, los distintos estratos de que se compone. El núcleo aparece como "el dominio en que la operación constitutiva se lleva a efecto" (pág. 231). Pero, en tanto que el nóema se refiere, por su sentido, al objeto, es menester un análisis más preciso del sentido y de la operación de dar sentido. Para ello, hay que volverse a la proposición, considerada como unión del sentido con las posiciones noéticas. La vuelta que efectúa la investigación revela la agudeza de la interpretación. El análisis del nóema mismo nos lleva de la mano al estudio del papel fundamental que juegan las posiciones noéticas en la constitución objetiva. Toda la exposición desemboca asi en el tema decisivo: el carácter constituyente de los actos ponentes. Su importancia resalta debidamente gracias al punto de partida elegido, el estudio del nóema, que obliga a dar este sesgo decisivo. Tal es el tema del capítulo más importante.

El Capitulo III trata exclusivamente de una región constituída, única de que se ocupan con detención las Ideas: la región del ser real. El acto constituyente del objeto real es la evidencia. En ella se unifican la posición y el ente dado (pág. 267). Algunas páginas, a modo de paréntesis, se dedican a distinguir entre las diversas acepciones de lo "real" y lo "existente". Se procede luego al análisis de la evidencia. La evidencia corresponde, por una parte, a un acto "vidente" y, por la otra, al carácter de ser "dado originariamente". Este último no depende del mero sentido del correlato intencional noemático, sino de los "modos de darse", en tanto que es un "darse originariamente". En él se legitima la posición vidente y-de modo correlativo - el darse originariamente del ente real se funda en el acto vidente. Mayz Vallenilla se enfrenta aqui con la ambigüedad que implica toda constitución; más adelante habremos de volver sobre ella. Los últimos parágrafos están destinados a mostrar cómo corresponde a cada acto vidente un modo distinto del "darse originariamente" y, en consecuencia, una distinta región del ente. Encontramos una de. terminación de los distintos tipos de evidencia de primordial utilidad para toda lectura de Husserl. Conclusiones: el acto de evidencia asertórica constituye la existencia contingente; la evidencia apodíctica otorga existencia universal y necesaria y versa sobre predicables de índole esencial; la evidencia adecuada funda el ser real absoluto y la inadecuada el ser real "cuestionable". Puesto que lo trascendente es objeto de una evidencia inadecuada, nada trascendente puede pretender realidad absoluta y el ser absolutamente cierto se encuentra reducido a la esfera de la inmanencia.

La investigación toca de continuo una ambigüedad inherente a la doctrina de la constitución en Husserl. La eviden. cia es constituyente de su objeto; por ende, es fundamento de su correlato. Esto debe interpretarse, creemos nosotros, en el sentido de la intencionalidad: la constitución no es "creación" del objeto por el acto ni, tampoco, proceso de "información" de una materia previa. Lo constituído es, a la vez, dado por sí mismo (cf. Ideen, § 97); y puede decirse que el darse a sí mismo del correlato funda, en todos los casos, la 
legitimidad del acto ponente (cf. Ideen, \$136). ¿Se trata, pues, de una recíproca fundamentación? ¿ 0 , más bien, de una sola operación, indiscernible en sus elementos, que, desde la vertiente del sujeto se veria como acto ponente y desde la perspectiva del objeto como existencia dada?

El comentario de Mayz refleja fielmente esta ambigüedad. Al sugerir el problema, leemos frases como las siguientes: "sólo cuando éste 'Modo de darse' el 'Objeto' reviste la forma del 'darse originariamente'... es susceptible de provocar en la conciencia una síntesis de verificación evidente" (pág. 292) ; "sólo cuando el 'Sentido' está caracterizado plenamente por un Modo de darse 'originariamente', el Objeto puede servir de base para lograr una Evidencia" (pág. 293; el subrayado es nuestro). Aquí se sugiere cierta prioridad del "darse" y hasta alguna índole pasiva de la evidencia. Pero otras frases corrigen de inmediato esta impresión y muestran la vertiente contraria; por ejemplo, cuando se dice que los actos de visión "funcionan como fundamentos" del ser real, o que éste "tiene su fundamento en la conciencia comprobable racionalmente" (pág. 294). Más clara aún se presenta esta ambigüedad en las páginas 323 y 324: "Es la índole natural de estos Entes la que, en último término, determina que los tipos de Visión se diversifiquen...". "Nuestra tarea, pues, consiste aquí... en puntualizar ante todo el 'Modo de aparecer' característico de los Entes inmanentes y trascendentes, y ver. . . cómo tal 'Modo de aparecer' determina específicas variaciones en los Actos de Visión correlativos". En cambio, de inmediato, se afirma: "Justamente la parte final de la investigación será destinada a poner de relieve cómo en tales Estructuras de Visión diversificadas se fundamentan, respectivamente, Modos de Evidencia correlativamente diferentes" (subrayamos nosotros). Las primeras frases aluden a una primacía de la patencia del ente, la última a la prioridad fundante del acto de visión. La ambigüuedad no es del intérprete, sino de Husserl; resulta, empero, tan esencial que obliga a explicitarla.

Mayz Vallenilla no se plantea expre. samente el problema, pero da la im. presión de dirimirlo, de hecho, en un sentido peculiar: el de conceder prioridad al acto ponente. En los parágrafos últimos se insiste en fórmulas del tenor de la siguiente: "El Acto de construcción 'objetiva' que realiza la Con. ciencia no es otro que el de otorgar 'Existencia' al Objeto asi visto" (pág. 351). El autor no hace sino ratificar la tesis implícita en toda su investigación, favorable a la prioridad del acto constituyente sobre el darse de lo cons. tituído. Pero cabría preguntar: ¿Es esta interpretación, sin más, evidente? Concediendo su justeza, ¿agota el significado de la constitución, o se limita a explayar uno de sus aspectos dejando en la sombra el segundo? ¿No derivará esta interpretación de un enfoque previo de la investigación?

Mayz Vallenilla insiste en que las Ideas están destinadas a fundar una crítica del conocimiento $y$, desde el principio, acepta que este criterio dirija su interpretación (págs. 22 y 64). Creemos justa esa opinión, con tal de no olvidar que la crítica del conocimiento sería al mismo tiempo, para Husserl, teoría del ser (del ser absoluto inmanente a la conciencia). Si el autor no hubiera olvidado la faceta ontológica de las Ideas, probablemente se habría percatado de la necesidad de plantear algunas preguntas capaces de añadir nueva luz a su problema. Por ejemplo: ¿Si lo constituído es dado, en qué consiste el carácter de lo dado en general? ¿Para que un ente sea patente es menester la previa posicionalidad de mi conciencia? $\mathrm{O}$, a la inversa: ¿Mi poder ser abierto al ente está determinado por la previa patencia de éste? A la luz de la crítica del conocimiento, la interpretación implícita en el libro de 
Mayz parece justa; a la luz de la ontología podría revelarse acertada, pero unilateral.

El libro que reseñamos constituye, más que una introducción, un comentario auténtico a las Ideas. Por su rigor y fidelidad resulta una guía segura en la difícil lectura de Husserl. Para nosotros, tiene, además, el valor de un síntoma: la filosofía latinoamericana empieza a abandonar el terreno de las improvisaciones fáciles para emprender un camino más arduo por generoso: el camino de la veracidad filosófica.

\section{LUIS VILLORO}

Les fondements logiques des mathémathiques, por Evert W. Beth. Gauthier-Villars, Paris, 1955.

El problema de los fundamentos de las matemáticas constituye una de las preocupaciones actuales más conspicuas de los matemáticos, los lógicos y los filósofos de la ciencia. Por ello, un número muy considerable de los libros - y, sobre todo, de los artículos-que se publican sobre estas disciplinas, se refiere a los distintos temas implicados por este problema fundamental. Pero, justamente por el gran interés que despierta y por la mucha actividad que se realiza en torno suyo, es difícil encontrar una obra que presente con acierto el problema entero. Tal es, por cierto, uno de los méritos de este libro del eminente lógico y matemático holandés, profesor Beth. Se trata de una obra de conjunto en la cual se encuentra una amplia información acerca de las aportaciones más recientes y de los diversos enfoques, aunada con una construcción sistemática sólida - que incluye varios desarrollos originales- y con una exposición clara y precisa.

El punto de partida general lo constituye el examen de la teoría de las ciencias de Aristóteles, ya que todas las concepciones modernas del problema coinciden en oponerse, de algún modo, a dicha teoría. En seguida se presentan concisamente los elementos básicos del análisis matemático, comprendiendo las definiciones por abstracción, el sistema de los números enteros, las dos tendencias antagónicas a la diferenciación y a la generalización que caracterizan a las matemáticas contemporáneas, la extensión del análisis a los otros sistemas de números, el razonamiento y la definición por recurrencia, la teoría del número natural y las consecuencias de la aritmetización del análisis. Luego, viene la exposición de las funciones elementales de la lógica simbólica. La lectura atenta de estas cuestiones y la hechura de los ejercicios propuestos por el au. tor, permiten adquirir el adiestramien. to técnico necesario para lograr una mejor comprensión de los temas que siguen.

En la teoría de la demostración se plantean algunos de los problemas primordiales de la matemática y de la ló. gica, como son la formación de las teo. rías deductivas contradictorias y de las no-contradictorias, la formalización axiomática de las teorías científicas, la independencia y la saturación de los sistemas de postulados, el problema de encontrar un procedimiento general para decidir si una expresión lógica constituye un teorema o no lo constituve, y las consecuencias matemáticas y filosóficas que implica la axiomatiza. ción de una teoría. El tratamiento de la sintaxis y de la semántica penetra en el conocimiento de las condiciones indispensables para el manejo riguroso de los símbolos lógicos, los resultados de la axiomatización de la sintaxis y de la aritmetización sintáctica, el análisis semántico de las teorías lógicas, las aplicaciones de la semántica a la metodología científica y la consideración del sistema de postulados de una teoría como una definición implicita de ella.

La interpretación de la existencia de las entidades matemáticas, que forma 